\title{
Measurement of gas temperatures in dust-free and dusty argon discharges
}

\author{
A Pikalev, M Pustylnik, C Räth and H Thomas \\ Deutsches Zentrum für Luft- und Raumfahrt e. V., Institut für Materialphysik im \\ Weltraum, 82234 Weßling, Germany \\ E-mail: Aleksandr.Pikalev@dlr.de
}

\begin{abstract}
.
Gas temperatures in an argon low-power capacitively-coupled rf discharge were measured by means of tunable diode laser absorption spectroscopy on $1 s_{5}$ metastable states. It was shown that the uncertainty of the measurements in dust-free plasma could be minimized to $\pm 1 \mathrm{~K}$. In the presence of dust, due to instabilities in the dust subsystem (e.g. dust density waves), the uncertainty of the temperature measurements increased several times. Nevertheless, an evidence of the gas temperature increase in the presence of dust was detected. This result suggests that the microparticle suspensions, immersed in the low-pressure plasmas, significantly modify the temperature fields of the neutral gas.
\end{abstract}

Keywords: dusty plasma, laser absorption spectroscopy, gas temperature, rf discharge

The paper is published as:

A Pikalev et al 2020 J. Phys. D: Appl. Phys. 53075203

DOI: 10.1088/1361-6463/ab59aa 


\section{Introduction}

Complex or dusty plasma is a medium containing ionized gas and micron-sized solid particles. It is used in basic research as a model system for the particle-resolved studies of generic condensed matter phenomena [1,2]. On ground, 3D complex plasmas are disturbed by gravity. That is why, microgravity experiments are so valued.

However, in microgravity other disturbing effects become important. For example, if there is a temperature gradient in a background gas, a microparticle experiences the thermophoretic force. This force is proportional to and directed opposite to the temperature gradient. For example, temperature gradients $\sim 1 \mathrm{~K} / \mathrm{cm}$ cause the thermophoretic force comparable with an axial electric force in a dc glow discharge positive column, which is used for the manipulation of microparticles in PK-4 setup [3]. Also, the force can play a role in a void (a dust-free region inside a dust cloud [4]) formation [5], and can be used to compensate gravity in ground experiments [6].

A related effect caused by the temperature gradient is the thermal creep - the gas flow appearing near a nonuniformly heated surface. The creep is induced in the direction of the temperature gradient along the surface. This phenomenon can produce vortices in microparticle suspensions $|7|$.

It is well known that microparticle suspensions can locally and non-locally modify plasma parameters (densities of electrons, ions and excited atoms, electron temperature and electric fields $[8[13]$ ), whose spatial distributions determine the distribution of heat sources in a discharge. In addition to that, the microparticles themselves represent the local heat sources since their surfaces absorb the electron and ion flows [14]. The microparticles can, therefore, modify the gas temperature distribution and thermophoretic forces. This issue is rarely taken into account in planning and interpretation of microgravity complex plasma experiments.

In the work by Rothermel et al. [6] the thermophoresis could compensate gravity for a relatively small amount of particles, but with the increase of the amount of microparticles, the suspension started loosing symmetry with respect to the midplane of the discharge chamber. The authors suggested that the microparticles modified the temperature gradient in the plasma. The effect could also be explained by a modification of the thermophoretic force in a dense microparticle suspension in comparison with the force for a single particle [15].

Calculations for a dc glow discharge in neon with pressure of 0.9 torr and a current of $3 \mathrm{~mA}$ showed that microparticle suspensions increase the gas temperature in the tube center by $\approx 2 \mathrm{~K}[16$. This estimation was conducted for the microparticle suspensions with radius of $8.25 \mathrm{~mm}$ (half of the tube radius) and densities of $1 \cdot 10^{11}-4 \cdot 10^{11} \mathrm{~m}^{-3}$. The grain radius was $2.55 \mu \mathrm{m}$. The effect was explained by a growth of the axial electric field in the presence of the microparticles. An attempt to detect this phenomenon by means of Fabry-Perrot interferometry of the discharge emission 17 was not successful due to insufficient accuracy of the method.

Gas heating in a rf discharge was discussed in [18]. It was suggested that the microparticles increase the gas heating, which could lead to significant thermophoretic forces. The gas temperature distribution in a rf discharge was simulated in [19]. According to the simulation the microparticles increased the gas temperature, but the effect was quite 
small.

Experiments 12 showed that the growth of dust particles in an argon-acetylene mixture was accompanied by a drastic (about $50 \mathrm{~K}$ ) drop of the argon gas temperature. Reference [10] reported the dust-induced growth of the gas temperature in the discharge center in argon and argon-acetylene mixtures. The achieved temperature gradient should have led to the thermophoretic forces comparable to electrostatic forces.

All this shows that the issue of the microparticle-induced modification of the temperature field in discharges is controversial, potentially important for the complex plasmas, and, therefore, requires careful investigation. In the present work, we propose and discuss a rigorous approach to the tunable diode laser absorption spectroscopy (TDLAS) measurements of gas temperatures in argon discharges. Since small $(\sim 0.1 \mathrm{~K} / \mathrm{cm})$ temperature gradients may play a role in complex plasmas, we took great care of characterizing the uncertainties. Uncertainties of the temperature measurements are rarely reported in literature although TDLAS is a common method of temperature measurement [20]. The most accurate measurements were in [8] (inaccuracy $2-5 \mathrm{~K}$ ) and 10.21 (inaccuracy about $10 \mathrm{~K}$ ). After minimizing the uncertainties, we compared the measurements in dust-free and dusty discharges.

\section{Experimental setup}

The experiments were conducted in the ground-based PK-3 Plus chamber [22], which was supplemented with a TDLAS system (see figure 1). The plasma was produced by means of a capacitively-coupled rf discharge. Two electrodes were driven in a push-pull mode by a sinusoidal signal with the frequency of
13.56 $\mathrm{MHz}$ and the discharge power of $0.2 \mathrm{~W}$. Argon fed to the chamber with 3 sccm gas flow was used as a working gas. The pressure was about $37 \mathrm{~Pa}$. We used melamine formaldehyde spheres with the diameter of $1.95 \pm 0.05 \mu \mathrm{m}$ as microparticles.

The PK-3 Plus setup allowed us to control the temperature gradient between the electrodes. The bottom electrode could be heated by an electric heater and the top electrode was cooled by a fan. We measured temperatures on the outer surfaces of the two chamber flanges with Tinytag Explorer data logger. Without heating, the temperature difference between the flanges was $<0.5 \mathrm{~K}$.

To measure temperatures at different heights, the beam from the Toptica DL Pro laser was divided into two beams which passed through the rf discharge. The width of the laser spectral line was less than $1 \mathrm{MHz}$, which is much smaller than the width of the Doppler-broadened absorption profile. The laser scanned the wavelength over a range around the $772.38 \mathrm{~nm}$ argon spectral line with the frequency of $10 \mathrm{~Hz}$. A small fraction of the laser light passed though a FabryPerot interferometer to control the wavelength scanning speed. Two absorption profiles as well as the interferometer fringes were detected with photodiodes. Signals from all photosensors were recorded with the LeCroy wavePro 725Zi oscilloscope.

To prevent the saturation broadening, the laser beams were attenuated with neutral density filters to the level about $1.5 \mu \mathrm{W}$. At these laser light powers, the measured absorption line width exhibited no power dependence. For comparison, the optogalvanic excitation of the heartbeat instability was observed with laser powers higher than $1 \mathrm{~mW}$ 23.

In principle, the absorption of the laser 


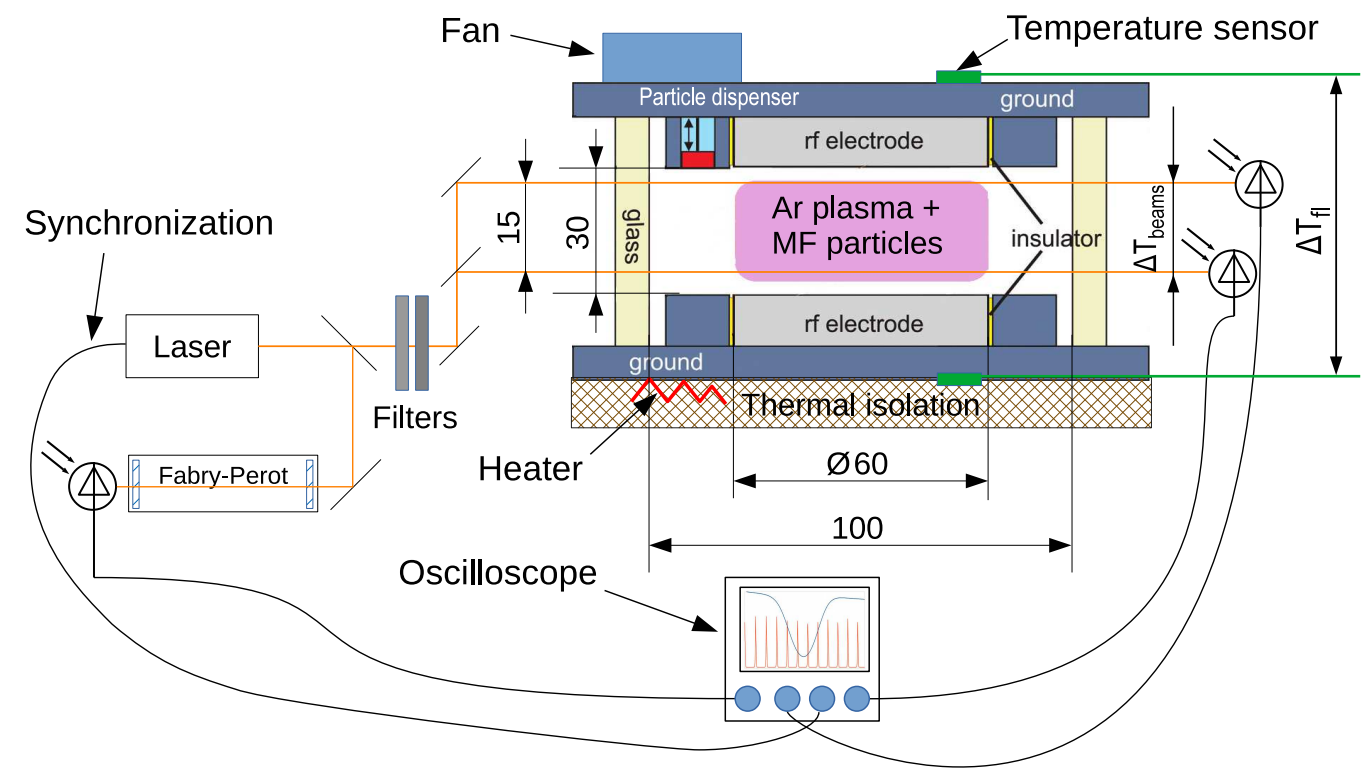

Figure 1. Scheme of the experimental setup. The heart of the experiment is the PK-3 Plus chamber 22. The bottom flange of the chamber can be heated to control a temperature gradient between the electrodes. The laser light is divided into two beams to measure the temperatures at two different heights. A fraction of the laser light passes through a Fabry-Perrot interferometer to control the wavelength scanning speed.

light by the microparticles could heat up their surfaces. If a microparticle absorbed the entire incident laser light, it would receive $4.5 \cdot 10^{-6} \mu \mathrm{W}$ of heat. Thermal flow density from the microparticle surface due to the heat exchange with the gas can be calculated as $J_{\text {gas }}=\frac{1}{4} N_{0}\langle v\rangle \alpha k_{B} \Delta T_{s g}\left[14\right.$, where $N_{0}$ - the gas number density, $\langle v\rangle$ - the average speed of the Ar atoms, $\alpha=0.86$ - the accommodation coefficient, $k_{B}$ - the Boltzmann constant, $\Delta T_{s g}$ - the difference between the surface and the gas temperatures. If we consider thermalization of the gas atoms on the surface as the only cooling mechanism, the heating by the laser will increase the microparticle surface temperature by less than $0.1 \mathrm{~K}$.

Therefore, we can assume that the temperature measurements we performed were practically non-invasive.

\section{Theory}

The TDLAS method is based on the BeerLambert law 24:

$I(\nu)=I_{0}(\nu) \exp (-k(\nu) l)$.

Here, $I_{0}(\nu)$ is the incoming laser intensity, $I(\nu)$ - the intensity after the absorption, $l$ is the length of absorbing medium and $k(\nu)$ - the absorption coefficient averaged along $l$. In our conditions, the absorption line had a Doppler shape:

$k(\nu) \sim \exp \left(-\frac{M c^{2}}{2 k_{B} T} \frac{\left(\nu-\nu_{0}\right)^{2}}{\nu_{0}^{2}}\right)$.

Here, $M$ is the atomic mass, $c$ - the speed of light, $T$ - the gas temperature, and $\nu_{0}$ - the spectral line center. It is assumed that the absorbing species have homogeneous temperature along the laser beam.

The absorption strength characterizes the 
metastable density:

$\int_{0}^{\infty} k(\nu) d \nu=h \nu_{0} B_{i k}\left\langle N_{i}\right\rangle$.

Here, $h$ is the Plank constant, $B_{i k}$ - the Einstein coefficient, $\left\langle N_{i}\right\rangle$ - averaged density of the absorbing atoms.

In every experiment, we measured two frequency-dependent intensities $I_{a b s}(\nu)$ and $I_{\text {laser }}(\nu)$ and two constant intensities $I_{\text {plasma }}$ and $I_{\text {dark }}$. By $I_{a b s}(\nu)$, we mean the intensity of the laser light passed through the plasma. This intensity contains the absorption profile. By $I_{\text {laser }}(\nu)$, we mean the laser intensity passed through the discharge chamber without plasma. $I_{\text {plasma }}$ is the plasma emission seen by the photodiode and $I_{\text {dark }}$ is its dark current. Since $I(\nu)=I_{a b s}(\nu)-I_{\text {plasma }}$ and $I_{0}(\nu)=$ $I_{\text {laser }}(\nu)-I_{\text {dark }}$, from equation (1), we obtain [21, 25]:

$k(\nu) l=\ln \left(\frac{I_{\text {laser }}(\nu)-I_{\text {dark }}}{I_{\text {abs }}(\nu)-I_{\text {plasma }}}\right)$.

\section{Data processing}

\subsection{Numerical procedures}

We aimed at measuring the temperature by fitting the experimentally measured profile from equation (4) with a Doppler profile from equation (2).

We have encountered two difficulties. First, the $I_{\text {laser }}(\nu)$ and $I_{a b s}(\nu)$ exhibited a small random phase shift with respect to each other (most likely) due to the weak interference of the laser light on the chamber walls. Second, in the presence of microparticles, the laser light experienced broadband extinction resulting in the reduction of $I_{a b s}(\nu)$ at unchanged $I_{\text {laser }}(\nu)$. Both effects are illustrated in figure 2.

To address both issues, we worked out a numerical procedure which matched $I(\nu)$ and

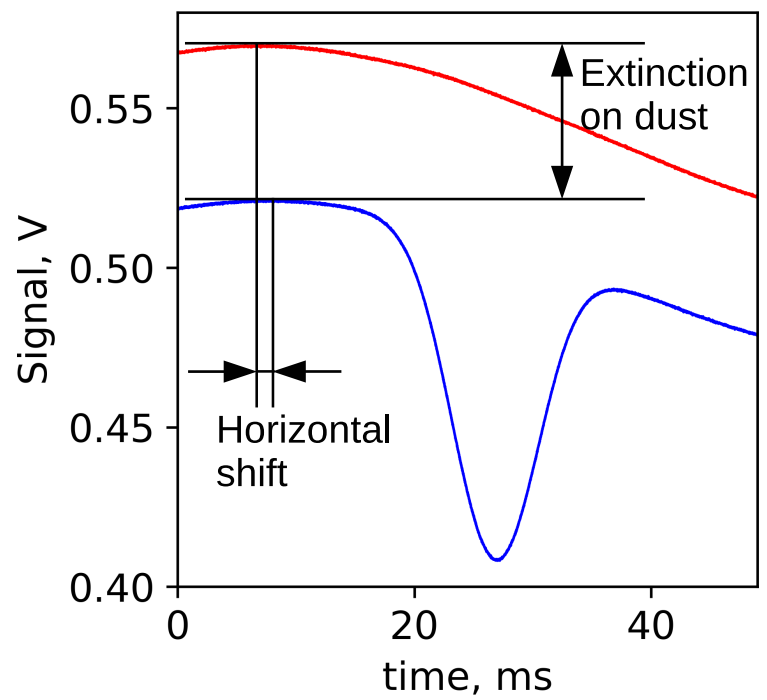

Figure 2. An example of absorption and laser intensity curves. The plasma emission and the dark signal are subtracted. The horizontal shift is $94.8 \mathrm{MHz}$. The microparticles extinguished $8.5 \%$ of the laser radiation.

$I_{0}(\nu)$ by minimizing the discrepancy $\Delta(\delta \nu)$ between points at the frequencies far from the spectral line center $\nu_{0}$. The function $\Delta(\delta \nu)$ was defined as

$\Delta(\delta \nu)=\left\langle\left(I(\nu)-s I_{0}(\nu-\delta \nu)\right)^{2}+w|\delta \nu|\right\rangle_{\left|\nu-\nu_{0}\right| \gg \Delta \nu}$,

where $I(\nu)=I_{a b s}(\nu)-I_{\text {plasma }}, I_{0}(\nu)=$ $I_{\text {laser }}(\nu)-I_{\text {dark }}, s$ characterizes the laser light extinction by the microparticles, $\delta \nu$ - the phase shift between $I_{\text {laser }}(\nu)$ and $I_{a b s}(\nu), w$ - arbitrary constant, $\Delta \nu$ is the spectral line width. We minimized this function using the golden-section method [26] calculating $s=$ $\left\langle I(\nu) / I_{0}(\nu-\delta \nu)\right\rangle_{\left|\nu-\nu_{0}\right| \gg \Delta \nu}$ at every iteration. We introduced the term $w|\delta \nu|$ to prevent unphysically large $\delta \nu$ in case of a very flat dependence of $I(\nu)-s I_{0}(\nu-\delta \nu)$ on $\delta \nu$, We took $w=5 \cdot 10^{-16} \mathrm{~V}^{2} / \mathrm{Hz}$ to make the term $w|\delta \nu|$ comparable with the noise for large shifts $\left(\sim 10^{8} \mathrm{~Hz}\right)$.

An example of the absorption fitted using the matching procedure described above is 
presented in figure 3. Discrepancy between the measurement and the fit is $<1 \%$.

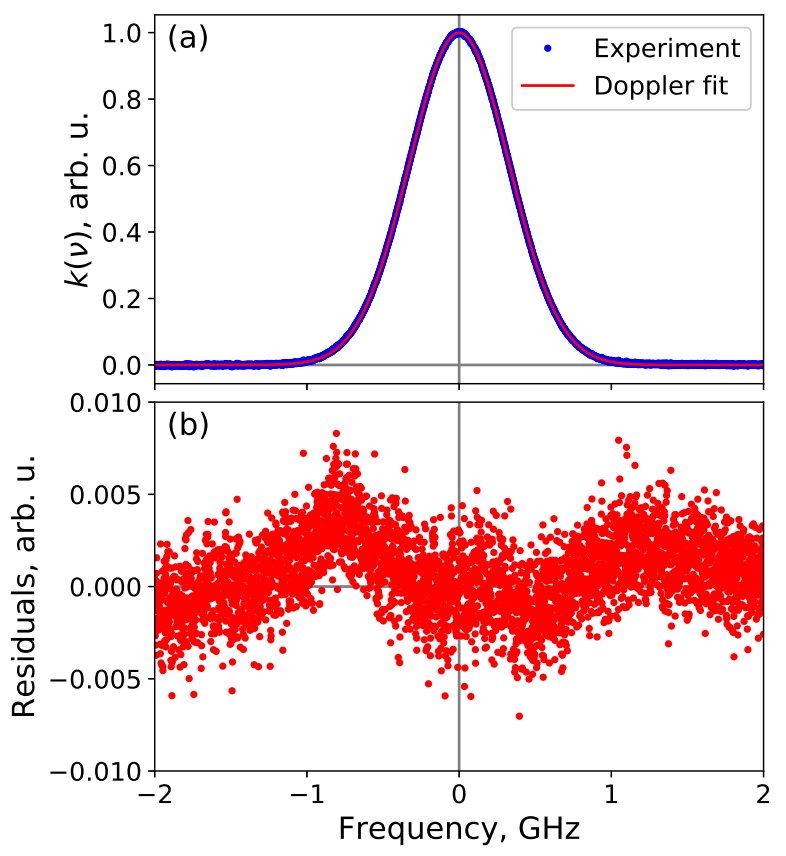

Figure 3. A normalized profile obtained from the curves shown in figure 2 by the matching procedure. (a) - the experimental points with the Doppler fit. (b) - the fit residuals.

Alternatively, the temperature could be found using the polynomial representation of $I_{0}(\nu)$ so that:

$$
\begin{aligned}
I(\nu) & =\left(a_{0}+a_{1} \nu+\ldots+a_{n} \nu^{n}\right) \times \\
& \times \exp \left(-A \cdot \exp \left(-b\left(\nu-\nu_{0}\right)^{2}\right)\right),
\end{aligned}
$$

where $A$ defines the absorption strength. The temperature can be found from the coefficient $b$ (see equation (2)).

This method, in principle, allows to avoid the above mentioned issues. To use this fit, we have to choose a proper $n$. Linear representation of $I_{0}(\nu)$ was used in [12], $n=2$ was used in [8], but in our case the laser intensity curves had more complicated shapes. Hence, we had to use a higher polynomial degree.

\subsection{Tests on artificial data}

We tested the data processing methods on artificially generated data. For the test, we used $T=305 \mathrm{~K}$ and absorption strength $A=$ 0.1 in the spectral line center. We generated $I_{a b s}(\nu)$ based on $I_{\text {laser }}(\nu)$ curves taken from real experiments using the following model:

$$
\begin{aligned}
& I_{a b s}(\nu)=s \cdot\left(I_{\text {laser }}(\nu-\delta \nu)-I_{\text {dark }}\right) \times \\
& \left.\times \exp \left(-A \cdot \exp \left(-\frac{M c^{2}}{2 k_{B} T} \frac{\left(\nu-\nu_{0}\right)^{2}}{\nu_{0}^{2}}\right)\right)+I_{\text {plas }(27 a)}\right)
\end{aligned}
$$

Phase shift $\delta \nu$ was randomly varied between -10 and $120 \mathrm{MHz}$. We generated random $I_{\text {dark }}=7 \pm 0.4 \mathrm{mV}$ and $I_{\text {plasma }}=$ $20 \pm 1 \mathrm{mV}$. The random values were uniformly distributed within these ranges. After that, we added Gaussian noise to the $I(\nu), I_{\text {plasma }}$ and $I_{\text {dark }}$. All parameters were chosen to be similar to the real experimental values. We performed the tests with 20 different $I_{\text {laser }}(\nu)$ curves taken from real experiments, and for every curve, we generated 100 tests.

The results of the comparison are presented in figure 4(a). We tested the fit (6) with $n$ from 2 to 6 . This fit with the polynomial degrees $n \leq 3$ gave very inaccurate results. Higher polynomial degrees gave uncertainties of about $\pm 3 \mathrm{~K}$. We also tested if the extinction of the laser light on the dust can affect the calculated temperatures. Introducing the factor $s$ of $0.8-0.99$ changed the calculated temperatures by less than $0.01 \mathrm{~K}$. The matching method showed an obvious advantage over the fits resulting in the uncertainty of about $\pm 1 \mathrm{~K}$.

In some cases, the measurements were conducted in the microparticle suspensions disturbed by the self-excited dust density waves (DDW) 27. This led to small modulations of the signals detected by the 

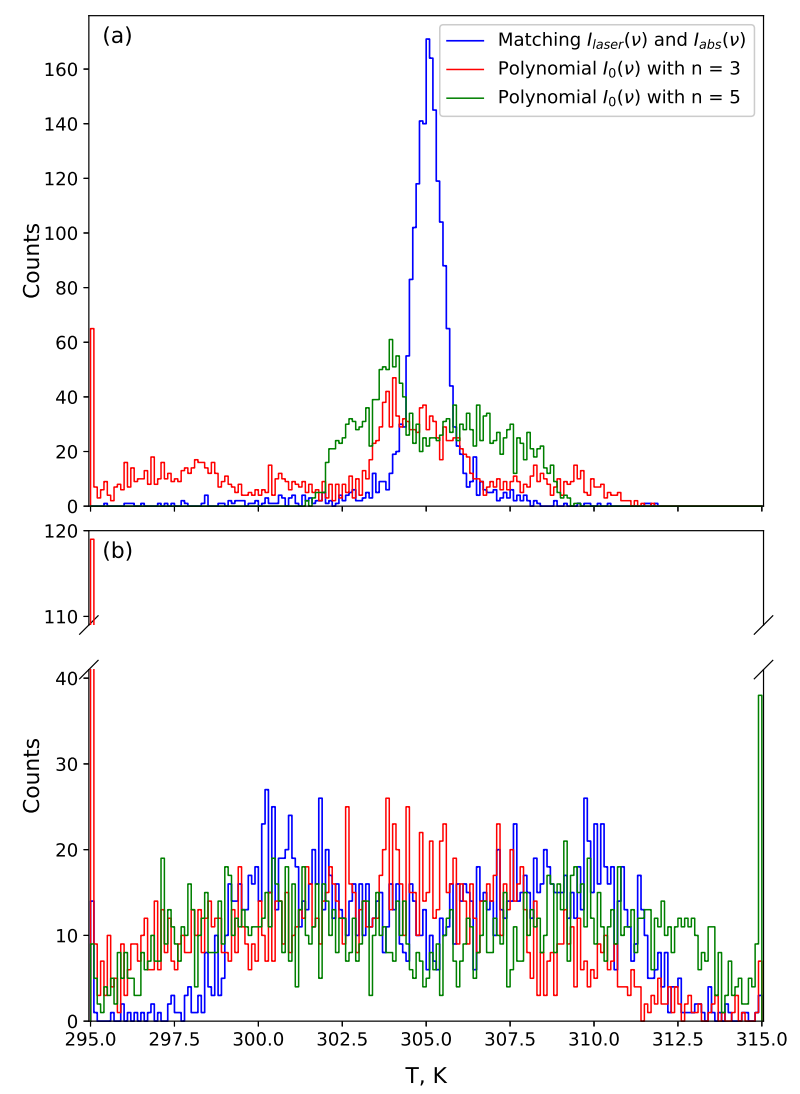

Figure 4. Distributions of temperatures determined from the artificially generated data by different methods (a) without and (b) with the disturbance caused by the self-excited dust-acoustic waves. The bins at the edges of abscissa accumulate also the results lying outside of the shown range. In both cases the matching $I_{\text {laser }}(\nu)$ and $I_{a b s}(\nu)$ brings best results.

photodiodes. The frequency of DDW was of the order of the scanning frequency. We included the effect of the waves into the model as sinusoidal signals with random frequencies, amplitudes and phases where the frequencies and the amplitudes were similar to those of the low-frequency noise we saw in the experimental signal. The results are presented in figure 4(b). Both data processing techniques resulted in a broad temperature distribution. Nevertheless, the matching technique produced less outlying results. For comparison, the matching technique gave errors more than $10 \mathrm{~K}$ only in 0.85 percent of the tests, while the polynomial fitting caused such spikes in 6.3 and 2.35 percent of the tests for $n=3$ and $n=5$ respectively.

Therefore, the matching technique showed a clear advantage under calm as well as under the DDW-disturbed conditions and was therefore used in the following analysis of experimental data.

\section{Results and discussion}

\subsection{Experiments without microparticles}

Temperatures measured using the top $\left(T_{t}\right)$ and the bottom $\left(T_{b}\right)$ beams are presented in figure 5. The data were obtained for different temperature of the chamber flanges $\left(T_{f l}\right)$, which was caused by the difference in the room temperature on different days. The measured temperatures increased with the the room temperature and were systematically higher than $T_{f l}$ by $5.4 \pm 0.9 \mathrm{~K}$.

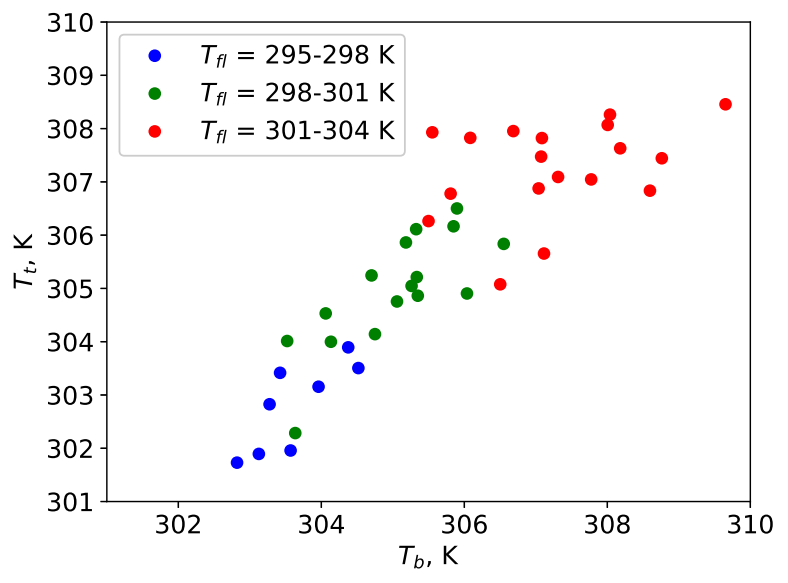

Figure 5. TDLAS-measured temperatures in a dustfree plasma at different temperatures of the chamber flanges. Different intervals of the temperature of the flanges $T_{f l}$ are colour coded. Since the temperature difference control was not used, the temperatures of the top and the bottom flanges differed less than by $0.5 \mathrm{~K} . T_{f l}$ varied due to the natural variation of the room temperature.

For additional testing, we conducted ex- 
periments with controlled temperature difference $\left(\Delta T_{f l}\right)$ between the flanges. The measured temperature difference $\left(\Delta T_{\text {beams }}\right)$ could be calculated in two ways: $T_{b}$ and $T_{t}$ could be determined independently and subtracted one from another and, alternatively, a ratio of the top and bottom absorption profiles $\left(k_{t}(\nu)\right.$ and $k_{b}(\nu)$, respectively) could be used. In the latter case, $\Delta T_{\text {beams }}$ was obtained by fitting

$\ln \left(\frac{k_{b}(\nu)}{k_{t}(\nu)}\right)=\frac{M c^{2}}{2 k_{B} \nu_{0}^{2}} \frac{\Delta T_{\text {beams }}}{T_{b} T_{t}}\left(\nu-\nu_{0}\right)^{2}+$ const. $(8)$

$T_{b}$ and $T_{t}$ in the denominator have to be obtained from the individual profiles $\left(k_{b}(\nu)\right.$, $\left.k_{t}(\nu)\right)$.

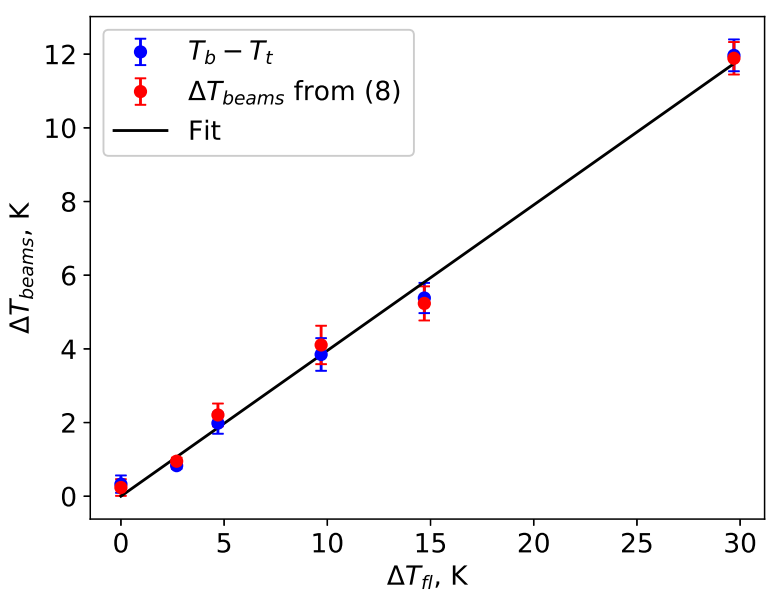

Figure 6. Dependence of the measured temperature difference $\Delta T_{\text {beams }}$ between the top and the bottom laser beams on the controlled temperature difference between the bottom and the top flanges. The values were obtained by averaging results from 3 to 13 experiments. The error bars show standard errors of the mean. The data are fitted by $\Delta T_{\text {beams }}=0.40 \cdot \Delta T_{f l}$

Both methods gave very similar results (see figure 6). $\Delta T_{\text {beams }}$ exhibited linear growth with $\Delta T_{f l}$, and could be fitted by $\Delta T_{\text {beams }}=$ $0.40 \cdot \Delta T_{f l}$. The slope of the line was somewhat smaller than the ratio of the interbeam and interelectrode distances.

\subsection{Effect of microparticles on the} temperature

We performed the experiments with the variable amount of the microparticles. In these experiments we did not use the control of the temperature difference between the chamber flanges. Small microparticle suspensions levitated in the bottom part of the chamber, whereas larger suspensions extended almost into the entire discharge volume (see figure 7). The microparticle number density was $\sim$ $10^{11} \mathrm{~m}^{-3}$. In some cases (e.g. as in figure 7(b)), the microparticle suspensions were disturbed by the DDW. Typical relative amplitude of the intensity oscillation due to the DDW was $\sim 10^{-4}$ after averaging over 100 oscillograms.

We used the fraction of laser light extinguished by the microparticles as a measure of their amount:

$E=1-\left\langle\frac{I_{a b s}(\nu)-I_{\text {plasma }}}{I_{\text {laser }}(\nu)-I_{\text {dark }}}\right\rangle_{\left|\nu-\nu_{0}\right| \gg \Delta \nu}$.

Here, averaging was performed over the points far from the spectral line center for which the absorption is negligible. These quantities $\left(E_{b}\right.$ and $E_{t}$ for the bottom and the top beam, respectively) determined in different experiments are shown in figure 8. In case of small suspensions, the top beam passed above the suspension (as in figure 7(a)). Larger suspensions extinguished also the upper beam, but to a lesser extent than the lower one.

To reduce the effect of the uncontrolled environment variations, we applied the measurement procedure schematically shown in figure 9. After stabilization of the laser and the discharge (several hours after switching on), we measured the absorption without the microparticles to determine $T_{\text {free } 1}$. Then we injected the microparticles, waited 5-10 minutes to avoid the measurement during the initial redistribution of the suspension and measured 

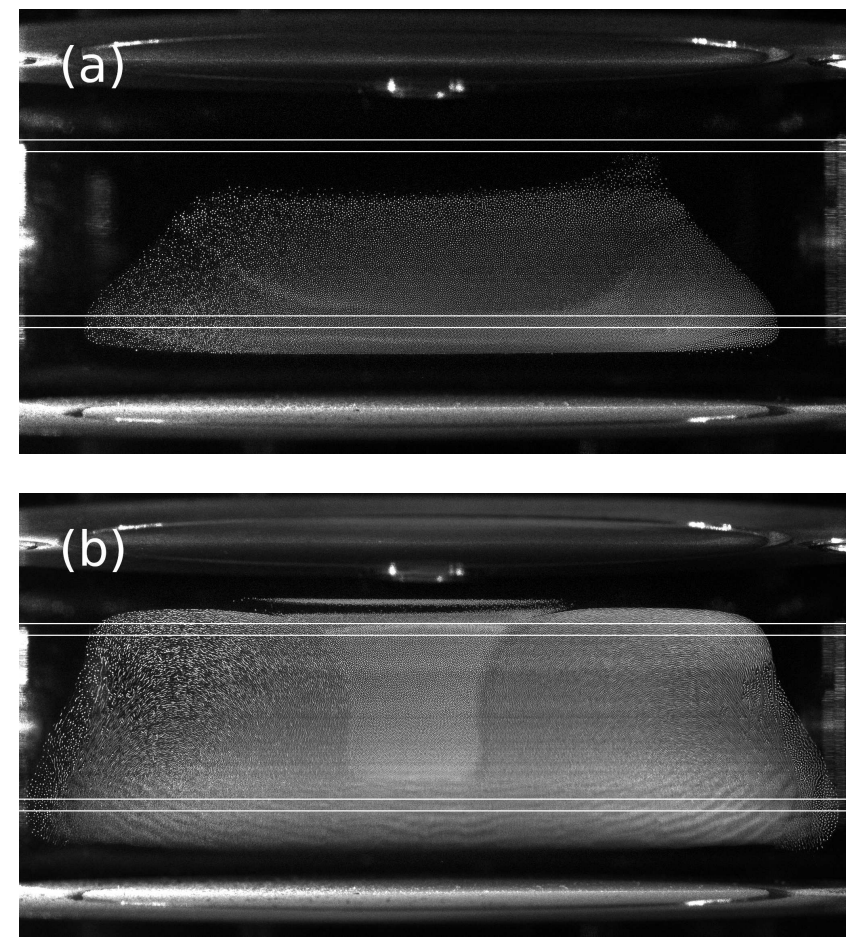

Figure 7. Images of (a) a small and (b) a large microparticle suspension used in the temperature measurement experiments. The horizontal lines denote the positions of the laser beams. In case (a), the laser beam passed the chamber above the suspension without crossing it. In case (b) the central part of the suspension is calm, whereas vortices are visible in the outer part of the suspension and DDW propagate in its bottom part.

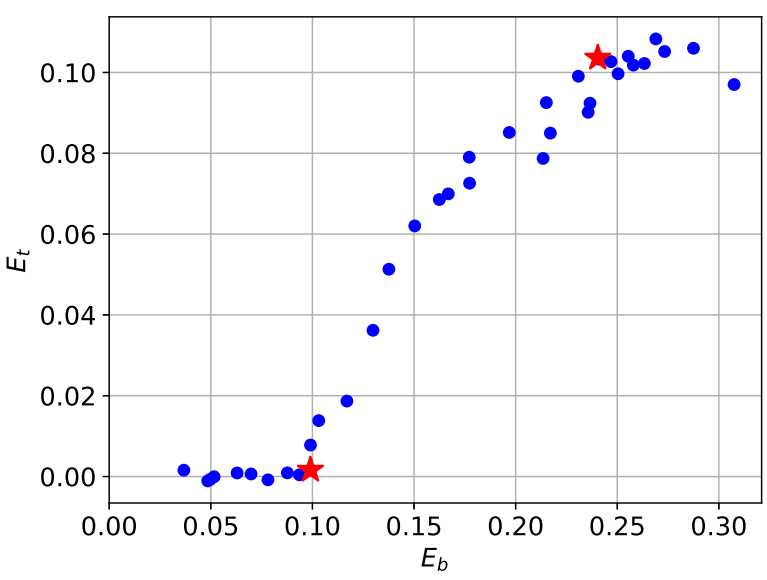

Figure 8. Fractions of laser light extinguished by dust in different experiments. The asterisks correspond to the suspensions in figure 7 .

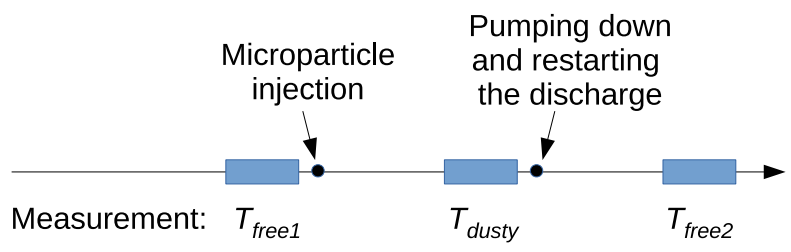

Figure 9. Experimental procedure for the determination of $\Delta T$ in equation 10 . To reduce the effect of the discharge condition instability, three gas temperature measurements were performed: two measurement in a dust-free discharge and, between them, one measurement in a dusty discharge.

$T_{\text {dusty }}$. During the measurement of $I_{\text {laser }}$ and $I_{\text {dark }}$, the discharge was switched off and the suspension was pumped out. After switching the discharge on again and waiting 5-10 minutes for its stabilization, we measured the absorption without the microparticles to obtain $T_{\text {free } 2}$. We repeated the procedure to obtain further data points using previous value of $T_{\text {free } 2}$ as next value of $T_{\text {free } 1}$. Each absorption measurement included the recording of all the signals from equation (4) and took 7-8 minutes. All absorption measurements were accompanied by measurements of the respective temperatures of the flanges $T_{f l \text { free } 1}, T_{\text {fldusty }}$, $T_{\text {fl free } 2}$.

Dust-induced temperature variation was calculated by

$\Delta T=\delta T_{\text {dusty }}-\frac{\delta T_{\text {free } 1}+\delta T_{\text {free } 2}}{2}$,

where

$\delta T_{\text {free } 1}=T_{\text {free } 1}-T_{\text {fl free } 1}$,
$\delta T_{\text {dusty }}=T_{\text {dusty }}-T_{\text {fldusty }}$,
$\delta T_{\text {free } 2}=T_{\text {free } 2}-T_{\text {fl free } 2}$.

The results are presented in figure 10 . The scatter in the resulting $\Delta T$ increased with the amount of microparticles and reached about $4 \mathrm{~K}$ at $E_{b}$ of about 0.25 . Nevertheless, figure 10 shows the evidence of the gas temperature increase in the presence of microparticles. 
This effect was predicted in 16 for the dc discharge in neon and explained by the increase of the longitudinal electric field in presence of dust. In our case, in a rf discharge, a similar mechanism could also be at work. In the presence of microparticles, the electrons are immobilized, which allows for the deeper penetration of $\mathrm{rf}$ electric field into the plasma [13, 28].

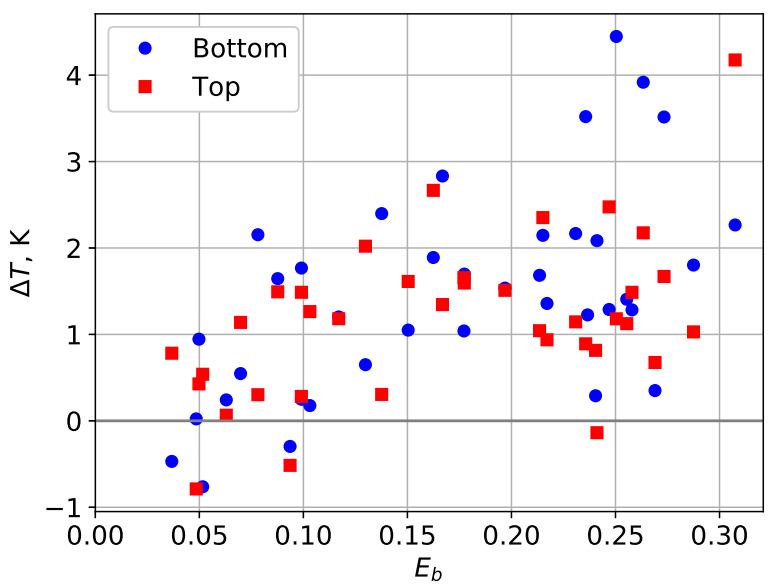

Figure 10. Dust-induced growth of the gas temperature. $E_{b}$ is used as a measure of the levitated dust amount.

The microparticle-induced modification of the spectral line absorption strength was calculated as

$R=\frac{2\left(k\left(\nu_{0}\right) l\right)_{\text {dusty }}}{\left(\left(k\left(\nu_{0}\right) l\right)_{\text {free } 1}+\left(\left(k\left(\nu_{0}\right) l\right)_{\text {free } 2}\right.\right.}$.

The results are presented in figure 11 . The presence of the microparticles always caused growth of the absorption for the top beam. On the other hand, small suspensions caused a decrease of the absorption for the bottom beam, whereas large suspensions induced the absorption growth.

Supposing no variation of the absorption length $l$ between dusty and dust-free cases, the results can be explained by a competition of such effects as electron depletion [12],

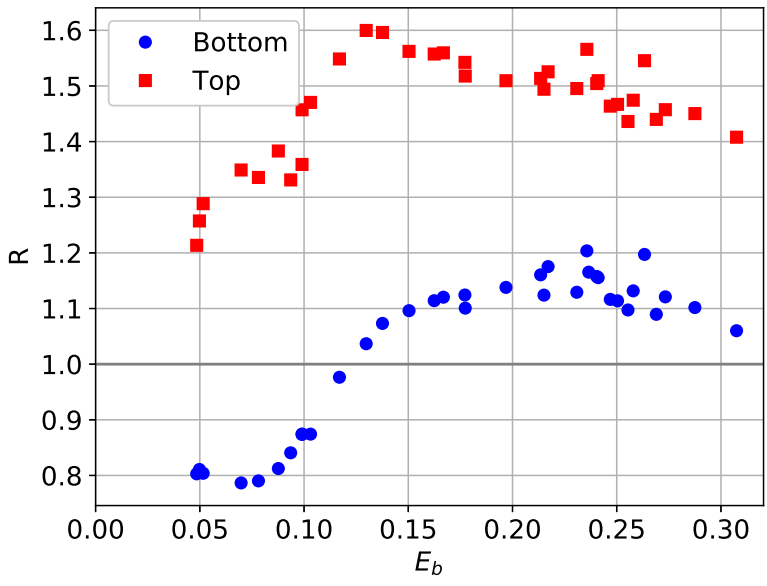

Figure 11. Relative microparticle-induced change of the absorption strength.

metastable quenching $[8]$ and electron temperature increase [10] in the presence of microparticles. Among those effects, the first two are of local nature, leading to the decrease of the metastable atom density, whereas the third one is non-local and therefore affects also the region where no microparticles are present.

In contrast to the metastable density, for the gas temperature, the effect of the microparticle cloud is always of the same sign for the top and the bottom beams. This suggests that the role of a local effect (absorption of electron and ion fluxes by the microparticles [14]) on the gas heating is negligible. The observed heating might be attributed to the global redistribution of the heat sources in the discharge [16].

\section{Conclusion}

We performed tunable diode laser absorption spectroscopy measurements of gas temperatures in an argon capacitively coupled rf discharge without and with microparticles at low power.

The measurements were performed with two laser beams at different heights simulta- 
neously. Without external heating, the measured temperatures were several $\mathrm{K}$ higher than those of the flanges. If we heated the bottom electrode, the measured temperature difference between the beams was proportional to the temperature difference between the chamber flanges.

The experiment showed that the presence of microparticles induced growth of the gas temperature. Heating was observed on both beams and vanished with the reduction of the microparticle amount. The metastable density exhibited different dust-induced modification: small suspensions caused reduction on the bottom beam and increase on the top beam, whereas, for larger suspensions, the increase was observed for both beams.

Spatial difference in the dust-induced modification of the gas temperature and metastable density allows to conclude about the underlying physical mechanisms. For the metastable density, the dust-induced modification is the result of the interplay between the local absorption of the metastable atoms and electrons by the microparticles and non-local increase of the electron temperature. For the gas temperature, it seems like the non-local redistribution of heat sources always dominates, whereas the local heat deposition due to absorption of electrons, ions and excited atoms on the microparticles seems to be negligible.

For further understanding, space resolved measurement techniques like laser-induced fluorescence or TDLAS with a laser sheet are required.

\section{Acknowledgments}

This work was supported by Deutscher Akademischer Austauschdienst (DAAD) with funds from Deutsches Zentrum für Luft- und
Raumfahrt e.V. (DLR).

The PK-3 Plus chamber was funded by the space agency of DLR with funds from the federal ministry for economy and technology according to a resolution of the Deutscher Bundestag under grant No. 50WP0203, 50WM1203.

We would like to thank Dr. V. Yaroshenko for careful reading of our manuscript and valuable suggestions.

[1] Morfill G E and Ivlev A V 2009 Rev. Mod. Phys. 81(4) 1353-1404

[2] Ivlev A, Löwen H, Morfill G and Royall C P 2012 Complex Plasmas and Colloidal Dispersions: Particle-Resolved Studies of Classical Liquids and Solids (Series in Soft Condensed Matter vol 5) (World Scientific Publishing Co. Pte. Ltd.)

[3] Pustylnik M Y, Fink M A, Nosenko V, Antonova T, Hagl T, Thomas H M, Zobnin A V, Lipaev A M, Usachev A D, Molotkov V I, Petrov O F, Fortov V E, Rau C, Deysenroth C, Albrecht S, Kretschmer M, Thoma M H, Morfill G E, Seurig R, Stettner A, Alyamovskaya V A, Orr A, Kufner E, Lavrenko E G, Padalka G I, Serova E O, Samokutyayev A M and Christoforetti S 2016 Rev. Sci. Instrum. 87093505

[4] Goree J, Morfill G E, Tsytovich V N and Vladimirov S V 1999 Phys. Rev. E 59(6) 70557067

[5] Balabanov V V, Vasilyak L M, Vetchinin S P, Nefedov A P, Polyakov D N and Fortov V E 2001 J. Exp. Theor. Phys. 92 86-92

[6] Rothermel H, Hagl T, Morfill G E, Thoma M H and Thomas H M 2002 Phys. Rev. Lett. 89(17) 175001

[7] Mitic S, Sütterlin R, Höfner A V I H, Thoma M H, Zhdanov S and Morfill G E 2008 Phys. Rev. Lett. 101(23) 235001

[8] Do H T, Kersten H and Hippler R 2008 New J. Phys. 10053010

[9] Do H T, Sushkov V and Hippler R 2009 New J. Phys. 11033020

[10] Stefanović I, Sadeghi N and Winter J $2010 \mathrm{~J}$. Phys. D: Appl. Phys. 43152003

[11] Sukhinin G I, Fedoseev A V, Antipov S N, Petrov O F and Fortov V E 2013 Phys. Rev. E 87 013101 
[12] Sushkov V, Herrendorf A P and Hippler R 2016 J. Phys. D: Appl. Phys. 49425201

[13] Pustylnik M Y, Semenov I L, Zähringer E and Thomas H M 2017 Phys. Rev. E 96(3) 033203

[14] Maurer H R and Kersten H 2011 J. Phys. D: Appl. Phys. 44174029

[15] Shumova V V, Polyakov D N, Mataybaeva E K and Vasilyak L M 2019 Phys. Lett. A 125853

[16] Polyakov D N, Shumova V V and Vasilyak I M 2014 Dig. J. Nanomater. Bios. 9 1249-1254

[17] Pikalev A A and Luizova L A 2014 Ukr. J. Phys. $59375-378$

[18] Klumov B A, Ivlev A V and Morfill G 2003 JETP Lett. 78 300-304 ISSN 1090-6487

[19] Akdim M R and Goedheer W J 2003 Phys. Rev. E 67(6) 066407

[20] Makabe T 2019 J. Phys. D: Appl. Phys. 52213002

[21] Hübner S, Sadeghi N, Carbone E A D and van der Mullen J J A M 2013 J. Appl. Phys. 113143306

[22] Thomas H M, Morfill G E, Fortov V E, Ivlev A V, Molotkov V I, Lipaev A M, Hagl T, Rothermel H, Khrapak S A, Suetterlin R K, Rubin-Zuzic M, Petrov O F, Tokarev V I and Krikalev S K 2008 New J. Phys. 10033036

[23] Pustylnik M Y, Ivlev A V, Sadeghi N, Heidemann R, Mitic S, Thomas H M and Morfill G E 2012 Phys. Plasmas 19103701

[24] Ochkin V 2009 Spectroscopy of Low Temperature Plasma (WILEY-VCH Verlag GmbH \& Co. KGaA)

[25] Kaupe J, Coenen D and Mitic S 2018 Plasma Sources Sci. Technol. 27105003

[26] Press W H, Teukolsky S A, Vetterling W T and Flannery B P 2007 Numerical Recipes: The Art of Scientific Computing (Cambridge, New York, Melbourne, Madrid, Cape Town, Singapore, São Paulo: Cambridge University Press)

[27] Schwabe M, Rubin-Zuzic M, Zhdanov S, Thomas H M and Morfill G E 2007 Phys. Rev. Lett. 99(9) 095002

[28] Killer C, Bandelow G, Matyash K, Schneider R and Melzer A 2013 Phys. Plasmas 20083704 